\title{
Determining whether surgeons perform thyroid fine-needle aspiration as well as radiologists: an analysis of the adequacy and efficiency of ultrasound-guided fine-needle aspiration performed by newly trained head and neck surgeons and radiologists
}

\author{
Jiaxin Wang ${ }^{1}$, Yanli Zhu ${ }^{2}$, Yuntao Song ${ }^{1}$, Guohui $\mathrm{Xu}^{1}$, Hao Yu ${ }^{1}$, Tianxiao Wang ${ }^{1}$, Bin Zhang ${ }^{1}$ \\ ${ }^{1}$ Key Laboratory of Carcinogenesis and Translational Research (Ministry of Education/Beijing), Department of Head and Neck Surgery, Peking \\ University Cancer Hospital \& Institute, Beijing 100142, China; ${ }^{2}$ Key Laboratory of Carcinogenesis and Translational Research (Ministry of \\ Education/Beijing), Department of Pathology, Peking University Cancer Hospital \& Institute, Beijing 100142, China \\ Contributions: (I) Conception and design: J Wang, B Zhang; (II) Administrative support: Y Zhu; (III) Provision of study materials or patients: Y Song, \\ G Xu, H Yu, T Wang; (IV) Collection and assembly of data: J Wang, Y Zhu; (V) Data analysis and interpretation: J Wang; (VI) Manuscript writing: \\ All authors; (VII) Final approval of manuscript: All authors. \\ Correspondence to: Bin Zhang. Key Laboratory of Carcinogenesis and Translational Research (Ministry of Education/Beijing), Department of Head \\ and Neck Surgery, Peking University Cancer Hospital \& Institute, 52 Fucheng Road, Beijing 100142, China. Email: docbinzhang@hotmail.com.
}

Backgrounda Ultrasound-guided fine-needle aspiration (FNA) cytology is a crucial diagnostic technique
used to assess thyroid nodules. In the past, ultrasound-guided FNA was performed mainly by radiologists.
However, many surgeons are increasingly being trained for this procedure now. In this study, we aimed
to compare the adequacy and efficiency of ultrasound-guided FNA performed by newly trained head and
neck surgeons with experienced radiologists in a single institution. We also assessed the malignancy rates in
nondiagnostic nodules and the differences between benign and malignant nodules.

Methods: This is a retrospective study. The data from patients who underwent ultrasound-guided FNA performed by surgeons or radiologists in two consecutive years were collected. Medical records, cytology results, and surgical pathology results were analyzed.

Results: During the study period, a total of 2,405 ultrasound-guided FNAs were performed on 2,163 patients. The head and neck surgeons and radiologists performed 1,132 and 1,273 ultrasoundguided FNA procedures, respectively. The nondiagnostic rate was $14.49 \%$ for surgeons and $15.40 \%$ for radiologists $(\mathrm{P}=0.533)$. There were no differences in patient age, gender, nodule size, and other sonographic characteristics between the groups of patients who were treated by radiologists versus surgeons. The median waiting time from biopsy appointment to performing ultrasound-guided FNA was 0 days for head and neck surgeons, and 6 days for radiologists $(\mathrm{P}<0.001)$. Of the 40 patients who had a repeat FNA or surgery, 19 (47.50\%) had a malignancy. Preoperative information about age, gender, operator, and characteristics of nodules did not predict the outcome of nodules with Bethesda category I.

Conclusions: The adequacy of ultrasound-guided FNAs performed by head and neck surgeons is similar to that of skilled radiologists, while surgeons are more efficient than radiologists. Nondiagnostic FNA reports should not be considered benign, and repeat FNA or selective surgical treatment is recommended.

Keywords: Biopsy, fine-needle; radiologists; surgeons; thyroid nodule

Submitted Nov 11, 2019. Accepted for publication Mar 12, 2020.

doi: 10.21037 /gs.2020.03.34

View this article at: http://dx.doi.org/10.21037/gs.2020.03.34 


\section{Introduction}

Thyroid nodules are a common clinical problem and the incidence of thyroid nodules has increased with the extensive use of thyroid ultrasonography. Cytological evaluation of samples obtained from ultrasound-guided fine-needle aspiration (FNA) is the most efficient method to determine the malignancy risk of thyroid nodules. However, scant cellularity or a bloody sample may be insufficient for accurate analysis. A nondiagnostic FNA report is difficult for both patients and clinicians because it can lead to repeated FNA, multiple physician visits, and ultimately, a delay in definitive treatment. Radiologists currently perform many ultrasound-guided FNAs in China. At the same time, a growing number of clinical endocrinologists, cytopathologists, and surgeons are being trained in ultrasound techniques and ultrasound-guided FNA. Surgeon-performed FNA is safe, accurate, and efficient. Because thyroid nodules are often referred for surgery, surgeon-performed FNA may potentially reduce waiting time for surgery. In this study, we aimed to compare the adequacy and efficiency of ultrasound-guided FNA performed by newly trained head and neck surgeons with experienced radiologists in a single institution. We also assessed the malignancy rate in nondiagnostic nodules and the differences between benign and malignant nodules.

\section{Methods}

\section{Subjects}

All consecutive patients who underwent thyroid ultrasoundguided FNA between March 2017 and March 2019 at Peking University Cancer Hospital \& Institute were included. Patients' demographic data, including age, gender, and type of surgery, were extracted from the medical records. Thyroid nodule variables retrieved from ultrasounds were extracted from Department of Radiology reports, and surgical pathology and cytological reports were obtained from the Department of Pathology databases. Operators with fewer than 300 completed FNA procedures are defined as newly trained operators. For this study, 13 radiologists experienced in FNA and 5 surgeons newly trained to perform ultrasound-guided FNA were included. The size of the tumor was defined as the largest diameter measured on ultrasound. Nodules were biopsied according to the American Thyroid Association (ATA) guidelines (1). Some nodules that were less than $1 \mathrm{~cm}$ in diameter but with clinical or sonographic features suggestive of thyroid cancer were also biopsied. To evaluate the operator's nondiagnostic rate over time, the procedures were subdivided into six consecutive groups of 50 procedures each. For each group, we recorded the nondiagnostic rate of each of the five surgeons.

\section{Ultrasound-guided FNA technique}

The GE Logiq E9 ultrasound scanner (USA) with a $12-\mathrm{MHz}$ linear transducer was used for localization of the target nodule and to assess its sonographic characteristics, including echogenicity, margin, calcification, vascularity, and shape. Patients were examined in a supine position with their neck lightly stretched. FNA was performed with a 22or 23-gauge needle under direct ultrasound visualization in two passes, with no immediate pathologic evaluation to assess adequacy. A syringe was used to expel the needle content onto a glass slide. Aspirated material smeared over the glass slides and preserved with alcohol fixation was finally prepared with hematoxylin and eosin stain. No complications were reported.

\section{Cytology report}

Two cytologists analyzed the slides and reported their results. Adequate samples should present at least six groups of 10 follicular cells to enable a cytological report (2). The ratio of nondiagnostic cytological reports from ultrasoundguided FNA performed by the radiologists or surgeons was the variable used to determine the adequacy for each group. Bethesda criteria included I (nondiagnostic or unsatisfactory), II (benign), III [atypia of undetermined significance or follicular lesion of undetermined significance, (AUS/FLUS)], IV (follicular neoplasm/suspicious for follicular neoplasm), V (suspicious for malignancy), and VI (malignant). Patients with suspicious or malignant cytology (Bethesda categories IV, V, and VI) were recommended to undergo surgery.

\section{Statistical analysis}

Data were analyzed using SPSS version 23.0 (IBM Co., Armonk, NY, USA). All continuous variables were reported as mean \pm standard deviation $(\mathrm{SD})$ or median $(\mathrm{Q} 1, \mathrm{Q} 3)$. Student's $t$-test or Mann-Whitney U-test was used to analyze quantitative variables, and the association between qualitative variables was assessed through the chi-square test or Fisher test. A value of $\mathrm{P}<0.05$ was considered statistically 
Table 1 Bethesda cytological categories of all nodules for radiologists and surgeons

\begin{tabular}{lccr}
\hline Bethesda categories & Radiologist, $\mathrm{n}(\%)$ & Surgeon, $\mathrm{n}(\%)$ & $\mathrm{P}$ value \\
\hline I & $196(15.40)$ & $164(14.49)$ & 0.533 \\
II & $202(15.87)$ & $119(10.51)$ & $<.001$ \\
III & $206(16.18)$ & $185(16.34)$ & 0.915 \\
IV & $30(2.36)$ & $37(3.27)$ & 0.175 \\
V & $167(13.12)$ & $104(9.19)$ & 0.002 \\
VI & $472(37.08)$ & $523(46.20)$ & $<0.001$ \\
Total & $1,273(100.0)$ & $1,132(100.0)$ & - \\
\hline
\end{tabular}

I (nondiagnostic); II (benign); III (atypia/follicular lesion of undetermined significance); IV (follicular neoplasm/suspicious for follicular neoplasm); V (suspicious for malignancy); and VI (malignant).

significant.

\section{Results}

A total of 2,405 ultrasound-guided FNA procedures were performed on 2,163 patients during the study period. There were 1,583 women $(73.19 \%)$ and 580 men, and the age ranged from 12 to 88 years (mean age, 51.63 years). Head and neck surgeons performed 1,132 ultrasound-guided FNAs on 978 patients, and radiologists performed 1,273 ultrasound-guided FNAs on 1,211 patients.

In total, 196 of 1,273 (15.40\%) ultrasound-guided FNA samples obtained by the radiologists were inadequate, compared to 164 of $1,132(14.49 \%)$ ultrasound-guided FNA samples obtained by the head and neck surgeons $(\mathrm{P}=0.533$, Table 1). The overall incidence of nodules that were suggested to undergo surgery (Bethesda categories IV, $\mathrm{V}$, and $\mathrm{VI}$ ) was $52.55 \%$ for the radiologists and $58.66 \%$ for the surgeons $(\mathrm{P}=0.003)$. A total of 90 nodules of all the 360 nondiagnostic nodules had no ultrasound images or reports from this institution, so these nodules without ultrasound images or reports were excluded in the subsequent analysis. Table 2 shows the homogeneity of both groups regarding patient age, gender, and the sonographic characteristic of the nodules. There was no difference between radiologists and surgeons in patient age, gender, nodule size, and other characteristics.

As head and neck surgeons were newly trained, we evaluated the nondiagnostic rate of surgeons over the number of FNA procedures each performed. Surgeon 1, surgeon 2, surgeon 3 , surgeon 4 , and surgeon 5 performed $531,276,185,93$, and 47 times of ultrasound-guided FNAs, respectively. With the increasing frequency of FNA, the average nondiagnostic rate of each group was $21.05 \%$, $16.06 \%, 14.00 \%, 14.07 \%, 10.00 \%$, and $9.21 \%$ respectively (Figure 1).

To compare the efficiency between head and neck surgeons and radiologists, we calculated the waiting time from biopsy appointment to FNA being performed. The median waiting time was $0[0,2]$ days for head and neck surgeons, and $6[2,9]$ days for radiologists $(\mathrm{P}<0.001$, Figure 2$)$.

Of the 360 nodules with nondiagnostic FNA, 8 underwent repeat FNA, 32 chose to have surgery without repeat FNA, while 320 did not undergo repeat FNA or surgery in this institution (Figure 3). In the 8 repeated FNA nodules, 2 nodules were Bethesda category I, 4 nodules were Bethesda category II, 1 nodule was Bethesda category III (AUS), and 1 nodule was Bethesda category VI. The diagnostic rate for repeat FNA in initial nondiagnostic nodules was $62.50 \%$. Of the 32 nodules in the nondiagnostic category that underwent surgery, 18 (56.25\%) were confirmed to have carcinoma which were all papillary. Of the 14 benign nodules, 2 were fibrotic nodules (FN), 4 were nodular goiter (NG), 4 were Hashimoto's thyroiditis (HT), and 4 were chronic lymphocytic thyroiditis (CLT).

Considering Bethesda category II as benign and Bethesda category VI as malignant in pathology, 18 were confirmed as benign nodules, while only 19 nodules were identified as malignant. The patient age, gender, operator, and characteristics of the nodules were then compared (Table 3). The mean nodule sizes were $1.21 \pm 0.76$ and $1.13 \pm 0.60 \mathrm{~cm}$ in the benign and malignant groups, respectively $(\mathrm{P}=0.753)$. The nodule characteristics of both groups were similar, including echogenicity, margin, calcification, vascularity, and shape. There were also no significant differences in patient age, gender, and operator. 
Table 2 Characteristic of nondiagnostic nodules

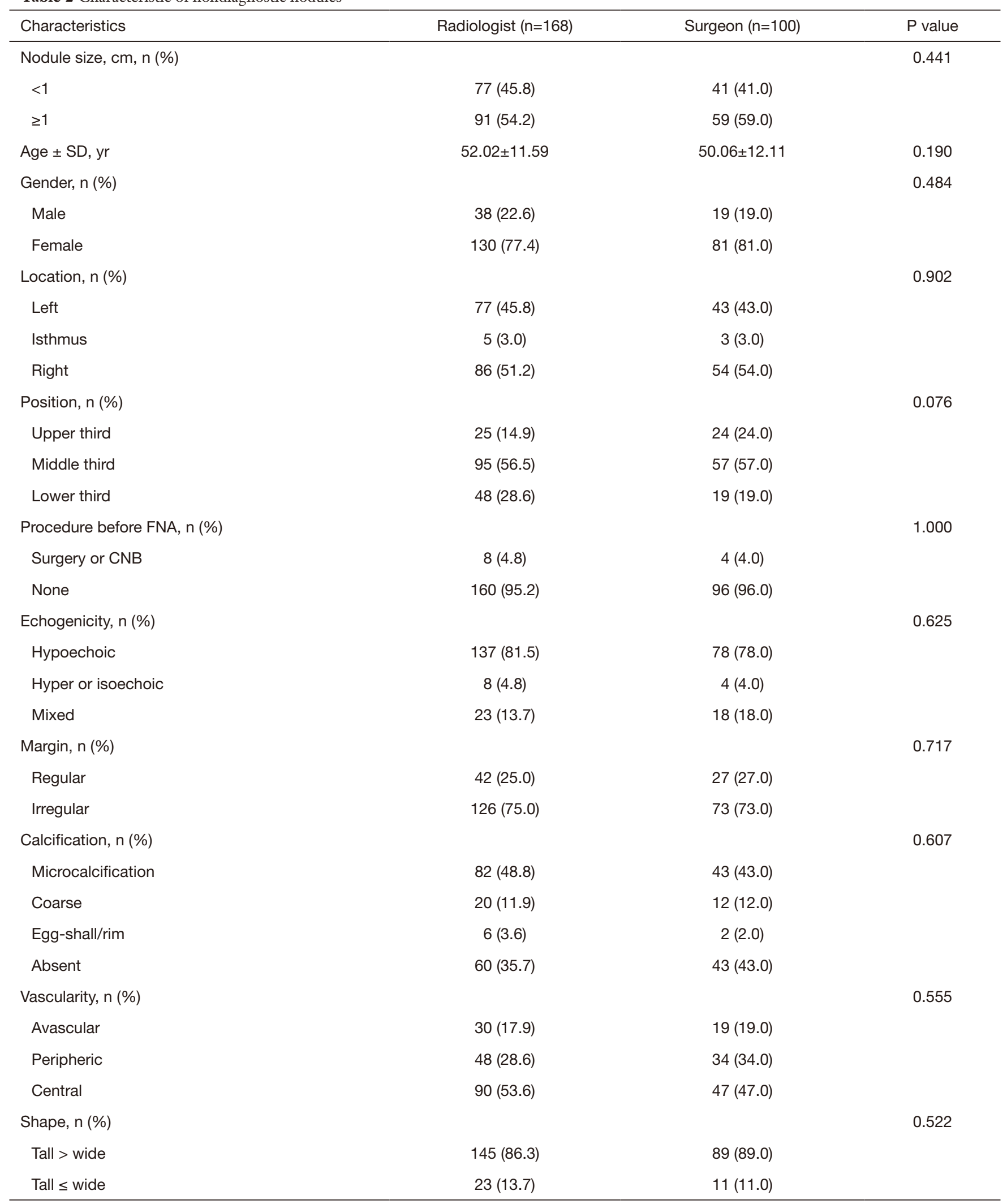

SD, standard deviation; FNA, fine needle aspiration; CNB, core needle biopsy. 


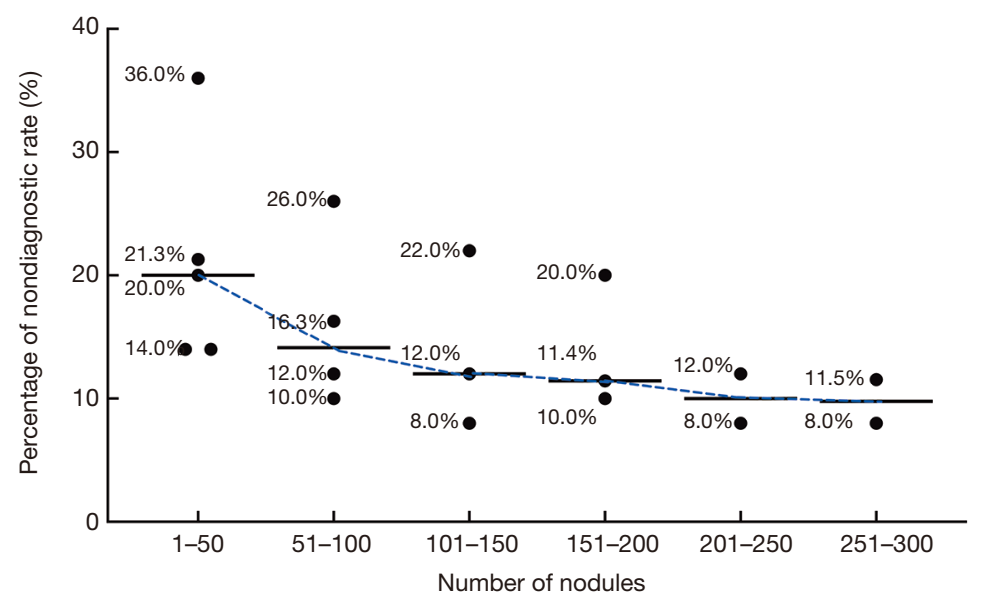

Figure 1 Nondiagnostic rate of surgeons over the number of FNA procedures each performed. FNA, fine-needle aspiration.

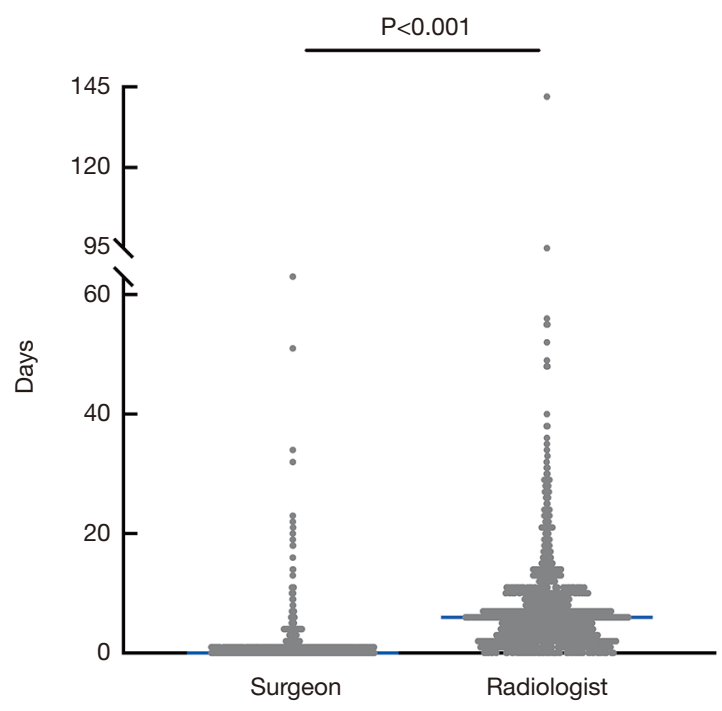

Figure 2 Waiting time from biopsy appointment to FNA being performed by head and neck surgeons and radiologists. FNA, fineneedle aspiration.

\section{Discussion}

Ultrasound-guided FNA biopsy is the most efficient and reliable means for the evaluation of thyroid nodules. A mean sensitivity and specificity rate of FNA as high as $88 \%$ and $90.5 \%$, respectively, has been reported (3). Although ultrasound-guided FNA is easy to perform, the nondiagnostic rate varies from $3.6 \%$ to $25.7 \%$ (4-8). Nondiagnostic cytological reports can undoubtedly result in a delay in diagnosis and definitive treatment. In our study, the overall nondiagnostic rate was $14.97 \%$, which is comparable to previous reports. Traditionally, thyroid ultrasound-guided FNA has been the domain of the radiologists, as ultrasound and ultrasound-guided FNA is a core competency acquired during radiology training. As patients with thyroid nodules are frequently referred to a surgeon for an opinion, more and more surgeons are trained to perform ultrasound-guided FNA in China. Ultrasound-guided FNA performed by surgeons provides a number of benefits, including assessment by a physician familiar with neck anatomy, reduction of multiple physician visits, and earlier triage to surgery if needed (9). However, there are few studies in the literature comparing radiologistperformed with surgeon-performed ultrasound-guided FNA in terms of adequacy and efficiency. This study found that the rates of adequate samples obtained by ultrasound-guided FNA performed by surgeons that were even newly trained were similar to those obtained by experienced radiologists. No differences in nodule characteristics were observed between radiologists and surgeons. Graciano et al. (7) also found that the adequacy rate obtained by surgeons newly trained in thyroid ultrasound was similar to that obtained by one experienced radiologist. These findings suggest that patients with suspicious thyroid nodules may be evaluated by head and neck surgeons who perform ultrasound-guided FNA as well as radiologists.

The multiplicity of variables related to procurement techniques that could not be controlled or measured precluded the determination of the precise cause for the differences. Numerous studies have assessed the degree to which different factors affect adequacy rates for thyroid FNA cytology. These factors include number of passes 


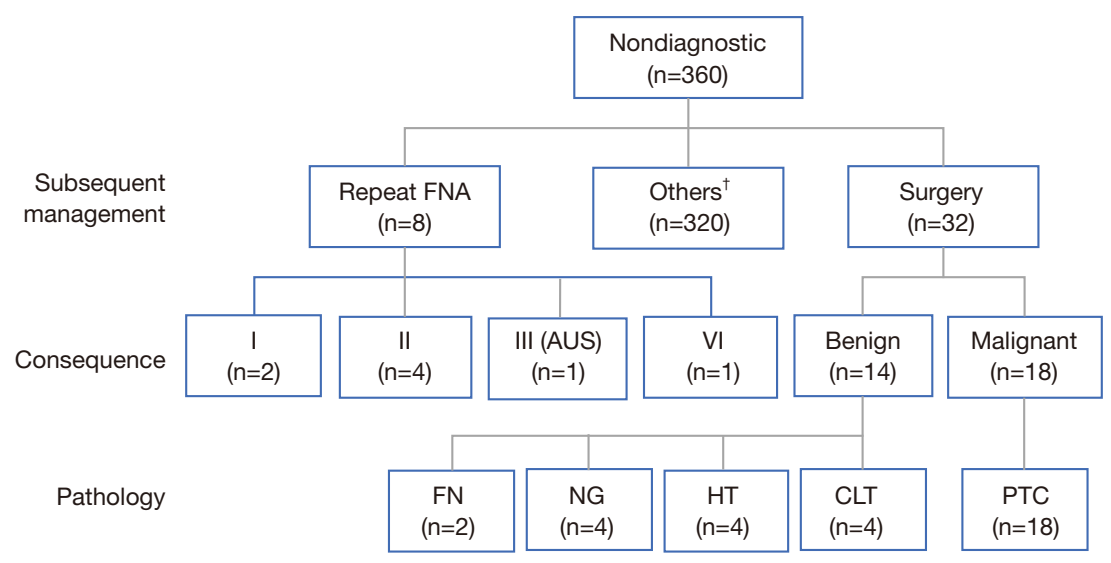

Figure 3 Management algorithm of nondiagnostic patients. ${ }^{\dagger}$, patients didn't undergo repeat FNA or surgery in this institution; I, Bethesda category I; II, Bethesda category II; III, Bethesda category III; VI, Bethesda category VI. FNA, fine-needle aspiration; FN, fibrotic nodules; NG, nodular goiter; HT, Hashimoto's thyroiditis; CLT, chronic lymphocytic thyroiditis; PTC, papillary thyroid carcinoma; AUS, atypia lesion of undetermined significance.

$(7,10)$, use of onsite evaluation $(11,12)$, nodule size or depth $(10,13)$, needle gauge $(14,15)$, cystic degeneration $(16,17)$, use of ultrasound guidance $(5,18)$, liquid-based cytology $(19,20)$, use of aspiration versus capillary technique $(21,22)$, and operator experience. However, it seems that in performing any procedure, the experience gained with time is no doubt of primary importance for successful clinical practice. De Fiori et al. (23) evaluated the nondiagnostic rates of 700 consecutive ultrasound-guided FNAs performed by a single radiologist and observed that the nondiagnostic rate was $32 \%$ for the first 100 cases, whereas it decreased to $13 \%$ for the following 200 cases. Recently, a study evaluated the outcomes of surgeons who performed ultrasound-guided FNA and found that the nondiagnostic rate was higher in early training (24). In our study, we also found that the average nondiagnostic rate of unskilled surgeons was high in the first 100 procedures $(21.05 \%$ and $16.06 \%)$. As expected, experience reduced the rate of nondiagnostic FNA in the following group, with an average nondiagnostic rate ranging from $10.00 \%$ to $14.00 \%$. Therefore, based on our first-hand experience, at least 100 procedures are required to ensure results in line with those reported in skilled radiologists in our institution and the literature. Since only surgeon 1 performed FNA more than 300 times, we showed the average nondiagnostic rate of the first 300 cases.

Although thyroid cancer has a favorable prognosis, most patients feel anxiety when a nodule is found in their thyroid. Patients appreciate the rapid diagnosis and management of medical problems. In this cancer institution, it usually takes about a week from when the clinic doctor orders a biopsy appointment to when ultrasound-guided FNA is performed by radiologists. However, most patients can receive ultrasound-guided FNA on the same day if the FNA is conducted by head and neck surgeons, which is very convenient for patients. Beyond that, patients can receive direct feedback from surgeons. Reeves et al. (25) found that the time from the first appointment to the time of a definitive management plan was 68 days shorter when FNA was performed by a surgeon rather than a radiologist. Moreover, surgeon-performed ultrasound-guided FNA for evaluation of thyroid nodules resulted in significantly lower costs. Another benefit of surgeon-performed ultrasoundguided FNA is the opportunity for surgeons to observe the shape, location, and size of thyroid nodules intuitively which is helpful for performing thyroidectomy.

According to the guidelines of ATA (1), repeated ultrasonography-guided FNA is recommended for thyroid nodules with initial nondiagnostic cytology. The diagnostic rate of repeat FNA in our series was $62.5 \%$ (5/8). Saieg et al. (26) also found that a repeat FNA in patients with initial nondiagnostic FNA could produce a diagnosis in the majority of cases. In their review of 86 nondiagnostic FNAs, 27 (31.3\%) remained nondiagnostic after repeat FNA. However, the malignancy rate of nondiagnostic FNA cannot be ignored. The malignancy rate of our surgical patients was $56.25 \%(18 / 32)$ which is significantly higher than that reported in other series which ranges from $2 \%$ to 
Table 3 Characteristic of nondiagnostic nodules confirmed by repeat FNA or surgery

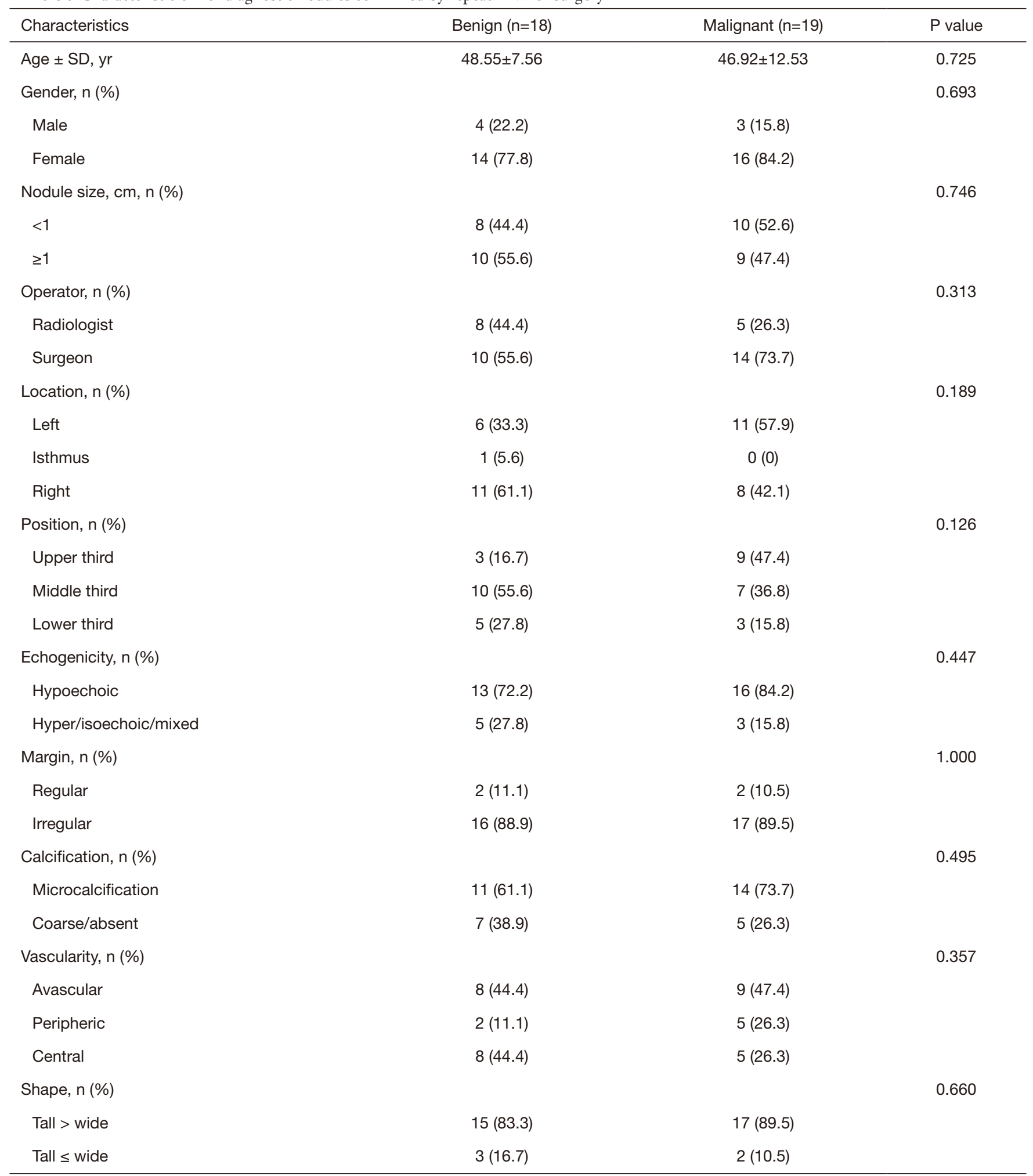

SD, standard deviation; FNA, fine needle aspiration. 
$37 \%$ (6,27-30). Considering Bethesda category II as benign and Bethesda category VI as malignant in pathology, the overall malignant rate of surgical patients and repeat FNA patients was $47.50 \%$ (19/40), which is still high. Our high malignancy rate likely reflects patient selection: patients with a higher suspicion of malignancy were more likely to be treated surgically or repeat FNA. Furthermore, most nondiagnostic FNA patients in this institution did not have a repeat FNA or surgery and were thus not included in the malignancy risk assessment. Sonographic features may be useful for identifying which nodules with repeat nondiagnostic FNA cytology results are more likely to be malignant. In the study of Moon, of the 104 nodules with 2 nondiagnostic cytology results, thyroid cancer was found in $25 \%$ of nodules with microcalcifications, irregular margins, a taller than wide shape, or hypoechogenicity, but was found in only 4\% lacking these features (31). Unfortunately, we have not found any characteristics of nodules that can suggest malignancy by comparing the characteristics of benign nodules with malignant nodules. Considering the malignancy risk of nondiagnostic nodules and the atypical features of ultrasound, repeat FNA followed by selective surgical treatment is recommended for patients who are anxious and have a higher suspicion of malignancy.

There are some limitations to our study. First, this was not a randomized study. Nodules treated by surgeons or radiologists were not randomized, so there might have been patient selection bias. Furthermore, only 40 of 360 nondiagnostic nodules underwent surgery or repeat FNA in this study. Selection bias might have existed, and a large prospective study is necessary to overcome this bias.

\section{Conclusions}

To our knowledge, this is the largest study of ultrasoundguided surgeon-performed thyroid nodule FNA performed to date, with outcomes comparable to those reported in series performed by other specialists. Patients with suspicious thyroid nodules may be evaluated by the head and neck surgeons who perform the ultrasound-guided FNA to the same satisfaction as radiologists, and more efficiently. We also found a relatively high rate of malignancy among nondiagnostic biopsies. Repeat FNA followed by selective surgical treatment is recommended.

\section{Acknowledgments}

We would like to thank Prof. Jianyu Rao (Department of Pathology and Laboratory Medicine, UCLA, David Geffen School of Medicine, Los Angeles, United States) for suggestions on manuscript revision.

Funding: None.

\section{Footnote}

Conflicts of Interest: All authors have completed the ICMJE uniform disclosure form (available at http://dx.doi. org/10.21037/gs.2020.03.34). The authors have no conflicts of interest to declare.

Ethical Statement: The authors are accountable for all aspects of the work in ensuring that questions related to the accuracy or integrity of any part of the work are appropriately investigated and resolved. All patients provided written informed consent for their information to be stored in the hospital database and used for research, and this study was approved by the Medical Ethics Committee of Beijing Cancer Hospital (approval number:2017XJS14).

Open Access Statement: This is an Open Access article distributed in accordance with the Creative Commons Attribution-NonCommercial-NoDerivs 4.0 International License (CC BY-NC-ND 4.0), which permits the noncommercial replication and distribution of the article with the strict proviso that no changes or edits are made and the original work is properly cited (including links to both the formal publication through the relevant DOI and the license). See: https://creativecommons.org/licenses/by-nc-nd/4.0/.

\section{References}

1. Haugen BR, Alexander EK, Bible KC, et al. 2015 American Thyroid Association Management Guidelines for Adult Patients with Thyroid Nodules and Differentiated Thyroid Cancer: The American Thyroid Association Guidelines Task Force on Thyroid Nodules and Differentiated Thyroid Cancer. Thyroid 2016;26:1-133.

2. Guidelines of the Papanicolaou Society of Cytopathology for the examination of fine-needle aspiration specimens from thyroid nodules. The Papanicolaou Society of Cytopathology Task Force on Standards of Practice. Diagn Cytopathol 1996;15:84-9.

3. Cibas ES, Ali SZ, NCI Thyroid FNA State of the Science Conference. The Bethesda system for reporting thyroid cytopathology. Am J Clin Pathol 2009;132:658-65.

4. Zheng B, Zarka MA, Chen C, et al. The largest CAP- 
certified Chinese reference laboratory experience with the Bethesda system for reporting thyroid cytopathology: correlation with histologic and BRAF data. J Am Soc Cytopathol 2018;7:16-21.

5. Conrad R, Yang SE, Chang S, et al. Comparison of cytopathologist-performed ultrasound-guided fine-needle aspiration with cytopathologist-performed palpationguided fine-needle aspiration: a single institutional experience. Arch Pathol Lab Med 2018;142:1260-7.

6. Reuters KB, Mamone MCOC, Ikejiri ES, et al. Bethesda classification and cytohistological correlation of thyroid nodules in a Brazilian Thyroid Disease Center. Eur Thyroid J 2018;7:133-8.

7. Graciano AJ, Fischer CA, Chone CT, et al. Efficacy of ultrasound-guided fine-needle aspiration performed by surgeons newly trained in thyroid ultrasound. Head Neck 2017;39:439-42.

8. Bhatki AM, Brewer B, Robinson-Smith T, et al. Adequacy of surgeon-performed ultrasound-guided thyroid fineneedle aspiration biopsy. Otolaryngol Head Neck Surg 2008;139:27-31.

9. Al-azawi D, Mann GB, Judson RT, et al. Endocrine surgeon-performed US guided thyroid FNAC is accurate and efficient. World J Surg 2012;36:1947-52.

10. Kavanagh J, McVeigh N, McCarthy E, et al. Ultrasoundguided fine needle aspiration of thyroid nodules: factors affecting diagnostic outcomes and confounding variables. Acta Radiol 2017;58:301-6.

11. de Koster EJ, Kist JW, Vriens MR, et al. Thyroid ultrasound-guided fine-needle aspiration: the positive influence of on-site adequacy assessment and number of needle passes on diagnostic cytology rate. Acta Cytol 2016;60:39-45.

12. Jiang D, Zang Y, Jiang D, et al. Value of rapid on-site evaluation for ultrasound-guided thyroid fine needle aspiration. J Int Med Res 2019;47:626-34.

13. Moon HJ, Kwak JY, Kim EK, et al. Ultrasonographic characteristics predictive of nondiagnostic results for fineneedle aspiration biopsies of thyroid nodules. Ultrasound Med Biol 2011;37:549-55.

14. Zhang L, Liu Y, Tan X, et al. Comparison of differentgauge needles for fine-needle aspiration biopsy of thyroid nodules. J Ultrasound Med 2018;37:1713-6.

15. Gümüş M, Cay N, Algin O, et al. Comparison of 21 and 27 gauge needles for determining sample adequacy in the aspiration biopsy of thyroid nodules. Diagn Interv Radiol 2012;18:102-5.

16. Cengic I, Tureli D, Altas H, et al. Effects of nodule characteristics on sampling number and duration of thyroid fine-needle aspiration biopsy: size does not matter, but cystic degeneration ratio does. Acta Radiol 2017;58:286-91.

17. Isaac A, Jeffery CC, Seikaly H, et al. Predictors of nondiagnostic cytology in surgeon-performed ultrasound guided fine needle aspiration of thyroid nodules. J Otolaryngol Head Neck Surg 2014;43:48.

18. Izquierdo R, Arekat MR, Knudson PE, et al. Comparison of palpation-guided versus ultrasound-guided fine-needle aspiration biopsies of thyroid nodules in an outpatient endocrinology practice. Endocr Pract 2006;12:609-14.

19. Kim SY, Kim EK, Moon HJ, et al. Combined use of conventional smear and liquid-based preparation versus conventional smear for thyroid fine-needle aspiration. Endocrine 2016;53:157-65.

20. Abraham TM, de las Morenas A, Lee SL, et al. In thyroid fine-needle aspiration, use of bedside-prepared slides significantly increased diagnostic adequacy and specimen cellularity relative to solution-based samples. Thyroid 2011;21:237-42.

21. Gill AS, Amdur R, Joshi AS. Importance of FNA technique for decreasing non-diagnostic rates in thyroid nodules. Head Neck Pathol 2018;12:160-5.

22. de Carvalho GA, Paz-Filho G, Cavalcanti TC, et al. Adequacy and diagnostic accuracy of aspiration vs. capillary fine needle thyroid biopsies. Endocr Pathol 2009;20:204-8.

23. De Fiori E, Rampinelli C, Turco F, et al. Role of operator experience in ultrasound-guided fine-needle aspiration biopsy of the thyroid. Radiol Med 2010;115:612-8.

24. Fernandes VT, Magarey MJ, Kamdar DP, et al. Surgeon performed ultrasound-guided fine-needle aspirates of the thyroid: 1067 biopsies and learning curve in a teaching center. Head Neck 2016;38 Suppl 1:E1281-4.

25. Reeves M, Patel R, Harmston C. Surgeon-performed ultrasound-guided fine needle aspiration of thyroid nodules is cost effective and efficient: evaluation of thyroid nodule assessment in a provincial New Zealand hospital. N Z Med J 2019;132:60-5.

26. Saieg MA, Barbosa B, Nishi J, et al. The impact of repeat FNA in non-diagnostic and indeterminate thyroid nodules: a 5-year single-centre experience. Cytopathology 2018;29:196-200.

27. Park JH, Yoon SO, Son EJ, et al. Incidence and malignancy rates of diagnoses in the bethesda system for reporting thyroid aspiration cytology: an institutional experience. Korean J Pathol 2014;48:133-9. 
28. Chow LS, Gharib H, Goellner JR, et al. Nondiagnostic thyroid fine-needle aspiration cytology: management dilemmas. Thyroid 2001;11:1147-51.

29. MacDonald L, Yazdi HM. Nondiagnostic fine needle aspiration biopsy of the thyroid gland: a diagnostic dilemma. Acta Cytol 1996;40:423-8.

30. Thewjitcharoen Y, Butadej S, Nakasatien S, et al. Incidence and malignancy rates classified by The Bethesda
System for Reporting Thyroid Cytopathology (TBSRTC) - an 8-year tertiary center experience in Thailand. J Clin Transl Endocrinol 2018;16:100175.

31. Moon HJ, Kwak JY, Choi YS, et al. How to manage thyroid nodules with two consecutive non-diagnostic results on ultrasonography-guided fine-needle aspiration. World J Surg 2012;36:586-92.
Cite this article as: Wang J, Zhu Y, Song Y, Xu G, Yu H, Wang T, Zhang B. Determining whether surgeons perform thyroid fine-needle aspiration as well as radiologists: an analysis of the adequacy and efficiency of ultrasound-guided fine-needle aspiration performed by newly trained head and neck surgeons and radiologists. Gland Surg 2020;9(3):711-720. doi: 10.21037/ gs.2020.03.34 